\title{
Las identidades contemporáneas y el réquiem del Estado Nación
}

\author{
Juan Bautista Lucca
}

\begin{abstract}
Resumen
El presente articulo busca advertir sobre los resortes de un mundo contemporáneo, enrevesado en su complejidad, fluido en el desmantelamiento de sus firmes pasos previos, incierto en el devenir del presente, tortuoso en su vuelta al pasado, aterrorizado en el agigantarse del futuro. Estos aspectos ponen en tensión al sujeto, a aquel que grita a los vientos la duda acerca de su identidad y que recibe del eco una y otra vez la voz de su propia incógnita. Para ello, recorriendo las perspectivas de los teóricos sociales y políticos contemporáneos, nos interrogaremos sobre los alcances de las interpretaciones respecto de las identidades y la vitalidad del Estado Nación, para reconocer finalmente hasta dónde este último nos ofrece la seguridad ontológica que otrora requeríamos.
\end{abstract}

Palabras clave: Identidad; Estado Nación; Globalización.

Contemporary identities and the requiem of the Nation State

\begin{abstract}
The present paper wants to reconstruct the puzzle of our contemporary world, which is intricate in his complexity, fluid in his dismantlement, uncertain in theirs previous steps, tortuously in his return to the past, terrified of the future, aspects that in more than one opportunity they put in tension the identity of the subject, that one that shouts to the winds the doubt about his identity and that receives only the echo -again and again- of his own voice. So, in this paper, we will study about the scopes of these interpretations in relationship with the identities and the vitality of the Nation State to recognize, finally, if he offers us the ontological safety that once we were needing.
\end{abstract}

Key Words: Identity; Nation State; Globalisation.

\footnotetext{
${ }^{(*)}$ Doctor en Ciencias Sociales (FLACSO). Máster en Estudios Latinoamericanos por la Universidad de Salamanca. Diploma Superior en Pensamiento Social Latinoamericano y Caribeño (CLACSO) y Licenciado en Ciencia Política (Universidad Nacional de Rosario). Es profesor en Política comparada en la UNR e Investigador del Consejo Nacional de Investigaciones Científicas y Técnicas (CONICET). Miembro del Centro de Estudios Comparados de la UNR, Argentina. Mail: juanlucca@hotmail.com ORCID: https://orcid.org/0000-0001-9017-8619
} 


\section{Las identidades contemporáneas y el réquiem del Estado Nación}

\section{Introducción}

¿Qué debo hacer? No veo por todas partes más que oscuridad. ¿Creeré que no soy nada? ¿Creeré que soy Dios? Blaise Pascal

El presente artículo busca advertir sobre los resortes de un mundo contemporáneo, enrevesado en su complejidad, fluido en el desmantelamiento de sus firmes pasos previos, incierto en el devenir del presente, tortuoso en su vuelta al pasado, aterrorizado en el agigantar del futuro, aspectos que en más de una oportunidad ponen en tensión al sujeto, a aquel que grita a los vientos la duda acerca de su identidad y que recibe del eco una y otra vez la voz de su propia incógnita.

Es decir, nos interesa poder partir de la duda del epígrafe de Pascal, en el cual, carcomido por la oscuridad de su presente se interroga por su ser, sus contornos y las múltiples máscaras que puede usar ese yo, para luego preguntarnos dónde está el Estado Nación como referencia a nuestra duda ontológica.

Ahora bien, antes de llegar a pensar por qué es importante introducir la noción de identidad y qué queda del Estado, debemos ser capaces de señalar los diversos procesos que convergen en nuestro presente y que nos obligan a utilizar un prisma diferente a aquel del capitalismo organizado, la sociedad de masas e incluso el mundo moderno, aunque más no sea para encontrar los puntos de cesura en los cuales, al decir de Marx, lo sólido se desvanece en el aire.

Uno de los primeros cambios importantes de nuestra cotidianeidad refiere al tiempo, y a la sensación generalizada de que -retomando la frase de Hamlet- "the time is out of joint". Este dislocamiento del tiempo está emparentado con el diagnóstico recurrente de la instantaneidad del presente, volviéndolo una amalgama difícilmente aprehensible, que echa por tierra todas las certezas sobre la rutinización de nuestras vidas, tradiciones y seguridades.

En el panorama de los autores de esta segunda modernidad, modernidad tardía, ultra modernidad, mundo post, ya no es posible decir aristotélicamente que el tiempo es el número del movimiento respecto al antes y al después, porque la vorágine actual deslocaliza justamente el tiempo, lo hace frenético, carente de un momento de perspectiva para el antes y después; parece convertirse en el reino del momento, del presente continuo, de la levedad, de la fluidez, de la liquidez, de la incertidumbre, la suspensión de un tiempo sin historia (Fraser, 1992: 26). Esto los llevó a pensar si la aceleración del tiempo - y con ella la posibilidad de que la novedad se multiplique infinitamente- no conlleva la rotura final de los eslabones que engarzan la historia en términos modernos o en todo caso -como apunta G. Vattimo (1990: 15)- nos 


\section{Juan Bautista Lucca}

enfrentamos hoy a la ruptura de la temporalidad como una unidad, y por ende a la historia como un único camino.

A este carácter dinámico y voraz del tiempo debemos anexarle su desarraigo del espacio, en tanto dimensiones fundamentales a la hora de percibir, inscribir o construir quiénes somos a partir de tener resuelto cuándo y dónde somos aquellos que deci(di)mos ser (Giddens, 2001: 28; Bauman, 2003: 121). Si antaño el paese, aquel lugar en el cual nacíamos servía de norte orientador para comenzar a pensar nuestra identidad, esta brújula ha sufrido varios procesos que dificultan describir nuestra identidad entendida como la "pertenencia a...".

Este desarraigo es producto al menos de tres modificaciones del espacio como punto de pertenencia. En primer lugar, por la ruptura de la frontera entre lo público y lo privado (Arfuch, 2005: 41); en segundo lugar, por el surgimiento de espacios que, siendo públicos, están colmados de extraños o carentes de significación colectiva (centros comerciales, aeropuertos, etc.); y tercero porque se observa cómo se construyen nuevos espacios a partir de la virtualidad que, aunque son compartidos por semejantes, difícilmente sirvan para unir conscientemente a quienes lo comparten porque el nosotros que allí se configura es meramente tácito (Patron, 1998; Miguel, 2003: 132).

A este mundo dislocado, desarraigado y desanclado, pocas cosas parecen quedarle en pie, con lo cual el "triste y solitario final" termina siendo el lugar común de las reflexiones. Es por ello que autores como Ulrich Beck (1998:15) consideran que el vocablo post es la palabra clave de nuestro tiempo, pues denota la estancia en un paraje sin referencias, en el instante en que advertimos que el futuro llegó y todo aquello que se encuentra por delante ya no dispone de nombre.

Ahora bien, esta percepción evanescente, puede incluso referirse a los relatos comunes en los cuales coincidíamos en el pasado, tal como lo propuso Lyotard, al dictaminar el acabóse de las narraciones de la redención cristiana, el progreso, la ilustración, el espíritu hegeliano, la unidad romántica, el racismo nazi, el equilibrio keynesiano entre otros (Anderson, 1998: 47). Inclusive, pensando en el marxismo como relato, autores como Alain Badiou (1990: 37) afirman que el marxismo ha dejado de ser un lugar de inscripción, de saber y verdad, con lo cual "...mantenerse en el marxismo es ocupar un lugar destruido, y por tanto inhabitable".

Sin tiempo, sin espacio, sin relatos y parados en el borde del abismo, la pregunta por mis pares, la comunidad de referencia, aquellos con los cuales compartía la unión que hacía la fuerza de mi identidad se vuelve una incógnita recurrente. La respuesta a este cuestionamiento redunda una vez más en la soledad, o el monótono eco de nuestra propia voz, y la angustiosa sensación en la que nuestro mundo ha desaparecido y sólo nos queda retraernos al yo, tal como nos lo retrata Giddens en este fragmento: 
“...la modernidad quiebra el marco protector de la pequeña comunidad y de la tradición, sustituyéndolas por organizaciones más amplias e impersonales. El individuo se siente despojado y solo en un mundo donde carece de los apoyos psicológicos y del sentimiento de seguridad que le procuraban otros ambientes más tradicionales" (Giddens, 1995: 50).

Frente a este páramo desolador, la única vía de escape estaría en potenciar la mirada en la profundidad de nuestro yo interior que el mundo moderno nos había ofrecido, pero que la sociedad de masas parecía haber aplacado (Taylor, 1993:. 48). La búsqueda del yo individual (y por ende la tambaleante situación del yo colectivo) nos lleva en la actualidad hacia la ilusión del hombre autosuficiente, el aislamiento existencial, por el cual los sujetos no piensan ni se plantean proyectos más allá de aquello que supone su entorno inmediato, que es en el cual puede pesar su autonomía y por ende su poder individual. Tras este sombrío panorama de desolación individual, ¿por qué preocuparnos por nuestra identidad, si ella es justamente la que mejor demuestra el síntoma de la evanescencia de nuestro mundo? Es decir, ¿por qué preocuparnos por responder quién soy, si pareciera ser que solo he quedado para responderme a mí mismo esa pregunta?

\section{II}

“Destruimos solo lo que sustituimos” Augusto Comte

Frente a un mundo incierto, parece inevitable que la vuelta a un "nosotros", a nuestras identidades, sea una fórmula protectora de seguridad ontológica que nos ayude para revertir el pudor de la desnudez del ser (Bauman, 2003: 190). Sin embargo, esta labor es una tarea que roza muchas veces lo quimérico, ya que salimos en busca de nuestra identidad justo en el momento en que la hemos perdido; es decir, anhelamos -parafraseando a Milan Kunderanuestra pesadez sólo cuando no soportamos más la levedad que no nos aferra a nada.

Ahora bien, no debemos quedarnos únicamente con la noción de identidad entendida como la "pertenencia estable a..." (un espacio, un tiempo, un sector social, un relato, un sexo, una nación u otra comunidad de pares). Esta "pertenencia a..." puede tener un sustrato biológico como cultural, ya que uno u otro se convierte en un todo al cual nos arraigamos casi de una manera indefinida. Sin embargo, si detenemos la reflexión sobre las identidades en este punto, habría de cosificar la naturaleza y naturalizar la cultura, sin tener en cuenta que las identidades 


\section{Juan Bautista Lucca}

son la resultante de un proceso inacabado de construcción antes que un procedimiento de reproducción de algo dado en el cual el individuo o el colectivo poca influencia pueden ejercer para escribir su propia biografía (Chuche, 1996: 109).

Es por ello que nos interesa reconocer las nuevas características que se ponen en juego en la génesis de las identidades. De una forma esquemática, y sólo como una primera aproximación, consideramos que los trazos que marcan la morfología actual de las identidades aluden a su carácter multidimensional, volátil, relacional, conflictivo y diferenciador, entre otros. Los resabios de la asimilación esencialista, hacían de la identidad un recorrido biográfico unilineal y estático, en el cual la unidimensionalidad del hombre -al decir de Marcuse- era la escena de la parálisis, de la imposibilidad del cambio, de la presencia de masas zombis que no se preguntan por la complejidad del yo, presas en una jaula de hierro más pesada que la del propio Max Weber, dentro de un tejido sin costuras en el cual era imposible pensar el devenir del ser (Berman, 1988: 25). Si antaño un obrero nacía, vivía y moría como tal, hoy esa fotografía como único fragmento significante de nuestro ser es colocada en la dinámica de una secuencia fílmica, en la cual la biografía del yo adquiere incontables carices y múltiples dimensiones de significación.

Debemos pensar entonces la identidad como un proceso abierto y en construcción, que permita hoy reconocernos como obreros, mañana como defensores de la comunidad gay, y quién sabe más adelante, partícipes activos por la seguridad, o inclusive teniendo que dar cuenta de alguna de estas tres identificaciones en un mismo momento, o tener que priorizar una $u$ otra en instancias diferentes sin que por ello estemos abandonando nuestro yo a la esquizofrenia ontológica.

De esta manera, nuestras identidades serían piezas del rompecabezas en nuestra biografía (individual o colectiva) en la cual cada pieza tiene valor en sí misma, pues logra significar formas diferentes de vernos en el mundo. Sin embargo, difícilmente todas las piezas engarcen fácilmente para llegar a reconstruir esa figura única y total que parece haberse roto, sino que nos propone una tarea continua de bricolaje, una construcción nunca acabada, abierta al cambio, las situaciones, nuestras prácticas, y las interrelaciones con los otros (Arfuch, 2005: 24). Es decir, pensar las identidades como un proceso de marchas y contramarchas, múltiples puntos de partida, pero no necesariamente algún punto de llegada, lo cual nos enfrenta a las incontables caras y caretas tras las cuales esconder nuestra duda ontológica, pero también advertir lo intercambiable, volátil y fluido del uso de las mismas.

Uno de los autores que con mayor ahínco advierte este continuo movimiento de los puertos donde amarrar nuestro ser es Zygmunt Bauman, quien a través de su adjetivo omnicalificativo "fluido" ha logrado retratar lo etéreo de nuestro mundo y de las identidades contemporáneas. 
Desde la perspectiva del sociólogo polaco, es la modernidad misma la que ha perdido su pesadez, su andamiaje y con ello todos los enclaves que vinculaban a los seres humanos a sus lugares de arraigo y por ende que modelaban sus identidades. Hoy, en palabras de Bauman (2005: 179) “...la construcción de la identidad se ha trocado en experimentación imparable”, es decir, estaríamos confinados al desanclaje de nuestras identidades, o en todo caso condenados a vivir la experiencia de la génesis constante de las mismas.

Así, hemos abandonado nuestro carácter sedentario a la hora de definir quienes somos, para lanzarnos a la aventura nómada y vagabunda en la que en cada tiempo, lugar y relación, estamos compelidos a preguntarnos -y respondernos- sin cesar quiénes somos. (Maffesoli, 2000: 41; Arditi, 2000: 104). Ahora bien, esta sensación de eterno comienzo que plantea Bauman, en una radicalización ad infinitum del argumento echaría por tierra la posibilidad de pensar las identidades mismas, porque no existiría representación alguna de aquello que somos, sino una continua presencia, irrepetible y única, que nos condena al mismo vacío del cual la problemática de la identidad busca salir.

Creemos que los extremos de la "pertenencia estable a..." y "la efímera y fluida adscripción a...”, es un callejón sin salida en el cual toda pregunta por quién soy, nos enreda en una maraña irresoluble (Cuche, 1996: 111). Sin embargo, la salida está en seguir el hilo de Ariadna, en primer lugar, haciendo hincapié en el carácter situacional de las identidades, para luego develar el carácter relacional de las mismas, ya que ello nos permitirá entrar sin perdernos en el laberinto. Focalizar en las situaciones de nuestra identidad supone evitar la encerrona esencialista, ya que las condiciones de nuestra existencia estarían marcadas por la contingencia de un mundo exterior y la interacción diferenciadora con(tra) otras identidades. Es por ello que autores como Renato Ortiz (1996: 78) apuntan con razón que “...no tiene mucho sentido la búsqueda de la existencia de "una" identidad; sería más correcto pensarla a partir de su intersección con otras identidades, construidas según otros puntos de vista". Los analistas de la identidad comparten la figura del otro como parte esencial en la definición del nosotros, ya que es justamente en el momento del encuentro de alguien diferente a nosotros que la pregunta por nuestra singularidad acaece.

En el encuentro con el otro está la clave de las perspectivas de las identidades contemporáneas, es decir el carácter dialógico y situado en toda constitución de identidades pues, como menciona Charles Taylor (1993: 55) “...el que yo descubra mi identidad no significa que yo la haya elaborado en el aislamiento, sino que la he negociado por medio del diálogo, en parte abierto, en parte interno, con los demás".

Ahora bien, este carácter contingente y relacional de las identidades no hace más que reflejarnos una vez más el carácter abierto de las mismas, ya que sin otra identidad que entre en disputa con 


\section{Juan Bautista Lucca}

la nuestra o sin cambios en nuestras condiciones de existencia, difícilmente estaríamos preguntando quiénes somos, ya que no viviríamos esa sensación de estar pisando sin el suelo. Asimismo, en el momento en el que nos encontramos con nuestro alter ya hemos dejado de ser el que éramos.

Estos son los esquemas habituales para comprender la identidad que han llevado, por ejemplo, a Ernesto Laclau (1997: 76) a asociar identidad y diferencia, ya que aquello con lo que me identifico no es solamente el propio contenido particular, "es también uno de los nombres de mi completitud ausente, el reverso de mi carencia original". En la propuesta de Ernesto Laclau (2000: 48), las identidades son un producto de las relaciones sociales, que encierran tras de sí una pugna por el poder, ya que en el mapa analítico de este autor todas las relaciones son de poder, lo que supone que todo intento por configurar una identidad dentro de este mundo contingente nos enfrenta a la disputa hegemónica, a la definición de las identidades como un acto político per se.

Otro aspecto que nos interesa recuperar de esta propuesta de Laclau, más compleja por cierto, es lo conflictivo de toda constitución de nuestras identidades, ya que no habiendo posibilidad ya de recurrir a esencias predeterminadas o valores absolutos previos (tierra, sexo, etnia, etc.) y estando en juego el poder en la definición de nuestra identidad, muchas veces el carácter agonal abandona la escena para que el antagonismo fuerce el contraste, haciendo que - parafraseando a Borges- ya no nos una el amor sino el espanto. Hay en esa frase del propio Borges una imagen condensada de la ontopolítica, es decir de la política de la identidad, que podemos discriminar por un lado como la ontopolítica de las similitudes frente a, por el otro, la ontopolítica de las diferencias.

La primera de ellas, está más ligada a los valores del universalismo original de la modernidad, que pone el énfasis en la igualdad entre todos los hombres, en aquello que nos trasciende más allá de nuestras singularidades, que podemos encontrar ejemplificada en la "Declaración del hombre y del ciudadano", donde naturalmente todos partíamos de un mismo inicio, y que se plasmó con gran potencia en la constitución del Estado Nación (al menos en su versión liberal clásica), como veremos más adelante.

La contrapartida a esta ontopolítica de la similitud es esta de la diferencia que veníamos retratando, que propugnó - por ejemplo- derechos de nueva generación para proteger esas diferencias - antaño en situaciones minoritarias y de discriminación negativa- inclusive con un criterio de discriminación positiva.

El problema que acarrearía esta nueva ontopolítica de la diferencia es dar status de identidad a algo que no es universalmente compartido. Entonces la pregunta reiterada a la cual las disputas por la identidad tendrían que dar cuenta es "cómo lograr la unidad en ( ¿a pesar de?) la 
diferencia y cómo preservar la diferencia en (¿a pesar de?) la unidad” (Bauman, 2005). Es allí donde el recipiente que mejor oficiaba de respuesta pacificadora - el Estado Nación- parece entrar en decadencia, y sin él parecen radicalizarse las fronteras, se agiganta el reverso de la diferencia y por ende se acrecientan todos los diagnósticos que una vez más, el hombre vuelva a ser el lobo del hombre.

\title{
III
}

\author{
"Tan pronto cuando alguien dice de los asuntos del Estado:-¿A mí qué me importa?- \\ hay que contar que el Estado está perdido" Jean-Jacques Rousseau
}

En este frenético presente, en el que aprehender nuestras identidades es una labor compleja que no se remite solamente a observar nuestras raíces, nuestra etnia, clase $u$ otro elemento que implique nuestro arraigo estable, la pregunta por la vida del Estado-Nación como articulador de nuestras biografías se ha convertido en una constante entre los analistas contemporáneos, especialmente al focalizar la incidencia de la "globalización" como proceso que en más de una oportunidad sería la causal de la muerte del Estado mismo y con ella el inicio de nuestras dudas ontológicas.

Aquí nos interesa repensar entonces cuáles eran los andamiajes en los que se asentaba la referencia al Estado Nación como articulador de nuestras identidades, para luego observar qué cambios habrían acaecido para que la sentencia de muerte tenga pretensión de validez, y finalmente interrogarnos sobre la morfología actual (o vacío si es que existe acta de defunción alguna) de las identidades vinculadas a los estados-nacionales.

Uno de los elementos centrales para ver la relevancia que otrora le asignábamos al Estado en nuestras definiciones respecto al ser, es la referencia a la tierra, a aquella parcela con fronteras que delimita nuestra pertenencia, un nosotros. En este sentido, tal como explicita el historiador Eric Hobsbawn (1996: 5-6) “...la identidad primordial que la mayoría de nosotros hemos elegido en este siglo XX es la del Estado Territorial, es decir una institución que establece un principio de autoridad sobre cada uno de los habitantes de un trozo del mapa".

Ahora bien, esta ligadura Identidad - Estado -Territorio - Soberanía que nos propone el historiador británico, suponía la mayor parte de las veces, la constitución de una monoidentificación, la pérdida de cualquier otro anclaje identificatorio (raza, paese, religión, dialecto, etc.) en pos de la unificación del hombre como ciudadano, alentando una suerte de homogeneización de las partes en el todo que conformaba la idea de nación (movimiento centrípeto que como vimos es un claro ejemplo de una ontopolítica de la similitud). 


\section{Juan Bautista Lucca}

Con estos criterios se construyeron los estados nación europeos, latinoamericanos y de posguerra, aunque el proceso de amalgama entre Estado y nación fue diferente en uno y otro caso, ya que -de forma anecdótica- los franceses, por ejemplo, tenían tantos quesos como naciones, Polonia de posguerra se dibujaría tras conocer censalmente dónde estaban los polacos y en la mayoría de los casos latinoamericanos la idea de nación fue una construcción misma del Estado.

Ahora bien, a pesar de la metáfora topológica en la cual se anclaba nuestra identidad en relación con el Estado y la Nación (y la preeminencia o preexistencia de uno u otro), la configuración de las identidades fue justamente eso, una construcción, la elaboración de una comunidad de destino a partir de la invención de símbolos (bandera, himno, héroes, etc.) y relatos, que galvanizaban una impronta histórica precisa y arbitraria, que es resignificada continuamente a través de la disputa por el sentido de la historia que le dan sus propios ciudadanos (Ortiz, 1996: 80; Aboy Carlés, 2001: 28 - 29 y 140).

Sin embargo, en la actualidad, este panorama parece entrar en colapso, y con él el Estado mismo, producto de los nuevos desafíos que se escudriñan tras la globalización todopoderosa. Un claro ejemplo de este retrato nos lo ofrece Habermas (2000: 12), cuando apunta que el problema reside en que “...la globalización fuerza al Estado-Nación a abrirse, en su interior, a una pluralidad de formas y vidas culturales nuevas y extrañas. $\mathrm{Y}$ al mismo tiempo restringe la capacidad de acción de los gobiernos nacionales en la medida en que fuerza a los Estados soberanos a abrirse a otros Estados".

En esta misma óptica, pero desde un contexto latinoamericano, autores como Aguilar Villanueva (1998, p. 48), proponen que la globalización impone al Estado Nación al menos cinco problemáticas para las cuales este no tenía respuestas efectivas y comprobadas, como son: “1) un mayor dinamismo de las corporaciones mundiales, 2) la formación de regiones económicas, 3) el surgimiento de regionalismos y las micro identidades colectivas, 4) la posibilidad de los conflictos entre civilizaciones y 5) el desarrollo de fuerzas sociales dotadas de mayor autonomía respecto del Estado".

De esta manera no es raro que encontremos numerosos estudios que hacen de estos presagios el inicio del fin, el puntapié para el dictamen de defunción del Estado Nación. Ahora bien, desentrañando estas lecturas sobre el advenimiento del fin del Estado, podríamos esquematizar su coincidencia en la influencia de dos tendencias disolutivas. La primera, que operativamente habremos de denominar Disolución desde arriba alude al incremento de la vulnerabilidad del Estado frente a los mercados globales y nuevos actores transnacionales.

Ello se ve reflejado con mayor claridad si tenemos en cuenta el presagio de James Anderson, quien aduce que actualmente estamos teniendo un cambio de percepción en la relevancia de las 
economías en el plano mundial como partes o etapas de periodización de la globalización. En un primer momento era evidente el dominio inglés y la presencia de economías estatales débiles, abiertas a la metrópolis. Luego, desde 1880 hasta la Segunda Guerra Mundial, ningún Estado dominaba el sistema mundial, mientras el proteccionismo crecía y el Estado se volvía centralizador de la economía. Después de la II Guerra Mundial, Estados Unidos tomó el rol de liderazgo mundial, para que en la década de 1970 la depresión económica mundial diera el puntapié inicial para una ola de apertura de las "economías nacionales" y para que las finanzas, la producción, el comercio y la economía estén más integradas y organizadas en una base global. (Anderson, 1995: 77).

A su vez, las estructuras decisionales y la soberanía estatal son sometidas a presiones desde arriba, no solo por la entelequia "mercado" sino también por nuevos actores, como son las instituciones supranacionales (por ejemplo el FMI, BM, G-8, etc.) que suponen ir más allá de las relaciones interestatales (que se dan principalmente en el campo diplomático-militareconómico entre Estados), para tener que hacer frente a relaciones transnacionales, que centran la mirada en otros segmentos de la realidad -como el campo económico, social, ecológico, cultural, entre otros- desde una óptica transfronteriza y aparentemente fuera de todo esquema territorial-soberano.

El otro movimiento disolutivo referido a los Estados nacionales es la Disolución desde abajo, que alude al incremento de la importancia de diferentes actores y agencias a nivel intra estatal. Es decir el surgimiento de la importancia de lo "local" en referencia a un "todo" que muchas veces se encarna en la figura del Estado Nación, que cada vez más articula elementos surgidos al interior de los Estados (ciudades, Regiones, etc.) (Anderson, 1995: 84).

En el plano de las identidades esta tendencia conllevaría al arraigo cada vez más en un ámbito territorial distinto al Estado Nación, como por ejemplo observamos en las reivindicaciones de autonomía de las regiones de Cataluña o Euskadi en España o bien la construcción (o bien recuperación) de un sustrato común de tipo continental, ya sea en su versión posnacional (Habermas, 2000) o en su regreso al sustrato histórico común, como propone para América Latina por ejemplo Alcira Argumedo (1993).

Ahora bien, creemos que si bien estas dos tendencias que venimos describiendo pueden servir la mayor de las veces como ilustrativas, existen algunos aspectos a desentrañar y morigerar, especialmente para pensar luego la vitalidad o no del rol del Estado Nación en la definición de nuestro ser.

El primero de ellos, recuperando las intuiciones de Anderson, lo llamaremos la Falacia de Gulliver. Recordemos que en este cuento británico su protagonista viaja en primer lugar a Liliput - un mundo donde todo mide la doceava parte que en su mundo, y donde entre sus 


\section{Juan Bautista Lucca}

habitantes reina el temor, la honestidad y la comunión- y en segundo lugar viaja a Brobdingnag - el reino de los gigantes que es doce veces mayor que el suyo y donde reina la codicia y el individualismo-. Parafraseando a Jonathan Swift, en la actualidad pareciera ser que el Estado ha encarnado a Gulliver, quedando siempre fuera de escala, desbordado por arriba o por abajo, dando lugar a que se generen nuevas micro identidades - es decir una vuelta al yo, que ha tomado el slogan small is beautifull - y una vorágine a escala global que nos confina a la soledad ontológica o bien a nuevas topografías ontológicas.

Ahora bien, focalizarse en el cambio de las escalas es llegar sólo a pensar en el nivel cuantitativo de los procesos políticos de una forma absoluta y cerrada; es decir, reemplazar a Gulliver por liliputenses o gigantes, dar rienda suelta a la aparición del Behemot - ya sea en su versión micro o macro- que venza finalmente al Leviatán o Estado.

Esta perspectiva disolutiva del Estado se ancla en otro elemento erróneo, que es imponer una lógica de suma cero a los espacios de significación (al menos en términos territoriales) de nuestras identidades. En este sentido, lo que tanto la disolución de arriba o de abajo suponen, es que más local o global significa menos Estado, y de cara a las tendencias actuales el Estado es cada vez menor, al borde de la inanición. Sin embargo, ello no supone su fin, sino más bien el inicio de nuevas complejidades a las cuales hacer frente. Ahora bien, a pesar de las presiones, creemos que el Estado sigue siendo uno de los códigos de acceso más importantes para nuestra significación identitaria incluso en el contexto globalizado" (Letourneau, 1997: 48).

Asimismo, tal como apunta Michael Mann (1997), estas tendencias (incluso la del fin del Estado) no toman en cuenta la diversidad existente de Estados, sus fortalezas y debilidades, la variabilidad del impacto globalizador, así como la metamorfosis que el Estado como entelequia histórico-intelectual ha venido sufriendo, entre otros aspectos. Aún en un mundo fluido, el anclaje al territorio sigue estando mediado por el monopolio del Estado, y por ende la identificación con la patria - a pesar de ser cada vez más dificultosa- sigue siendo funcional.

En términos de la construcción de las identidades, a pesar de la preeminencia que todavía mantiene el anclaje estatal, no podemos dejar de contrastar que fue la duda lo que mató al gato, es decir, fue la duda misma sobre la vida o muerte del Estado, lo que permitió aflorar todo un nuevo cúmulo de interpretaciones sobre la configuración de las identidades, en general ligadas a la ontopolítica de la diferencia. Estando en duda la fuerza centrípeta del Estado como significante de nuestras vidas, rápidamente las estratagemas centrífugas parecen haberse activado. Es decir, esta duda no abrió necesariamente las fronteras geográficas sino más bien simbólicas, posicionándonos frente a la pregunta problemática de la construcción de los sujetos no solamente en su sentido, sino también frente a otras alternativas geográficas (Yannuzzi, 1997). 


\begin{abstract}
"Senti, en la última página, que mi narración era un símbolo del hombre que yo fui, mientras la escribia y que, para redactar esa narración, yo tuve que ser aquel hombre y que, para ser aquel hombre, yo tuve que redactar esa narración, y así hasta el infinito. (En el instante en que yo dejo de creer en él,
\end{abstract}

"Averroes" desaparece).

Jorge L. Borges

En el punto final de la argumentación de este ensayo, creemos que nuestra reflexión antes de encontrar un punto de cierre, se enfrenta ante un punto de fuga, ante la sensación de "última página" tal como apunta el epígrafe de Borges. En un mundo en flujo, en un presente en cambio, con un Estado en duda, es inevitable que nuestras identidades sean el centro de las reflexiones, ya que podríamos decir en palabras de Bauman, que “...uno sólo investiga a fondo el valor de algo cuando se desvanece ante nuestros ojos, cuando desaparece o se desmorona"(Bauman, 2005: 101).

Ahora bien, este síntoma del desmoronamiento de nuestras identidades, produce la alteración del pentagrama de nuestra biografía, ya que si antaño la respuesta a la pregunta por el ser, el nosotros, el yo, se escribía con un tempo de blancas, en la actualidad esta se nos presenta como un allegro, con una dinámica rápida de corcheas y semicorcheas en el cual se suceden los Allegro molto - muy rápido-, Allegro ma non troppo - no demasiado rápido- o Allegro assai bastante deprisa-. En esta vorágine de notas, una de las formas de interpretación de quienes somos -que parecía estar más ligado al mundo de las notas blancas- como era el caso del Estado Nación, resulta una pausa abrupta, un descanso de mortuoria calma, en definitiva todo un réquiem para los tiempos que corren.

Sin embargo, entre la vorágine y la pausa eterna, la pregunta por las identidades adviene una y otra vez, traspasando la complejidad de los contratiempos y tiempos largos, para lo cual ni la parsimonia ni la prisa permiten aprender el proceso complejo de construcción de las mismas, para lo cual es necesario el conocimiento tanto de la levedad y la pesadez.

Es por ello que esta última página, el punto de fuga que proponemos para repensar las interpretaciones sobre las identidades supone la búsqueda de un tempo andante de interpretación, el tránsito por la tangente, en definitiva, abocarnos a retratar las identidades con el mismo método que nos propone Maquiavelo a la hora de hacerlo para la política de su época: 


\section{Juan Bautista Lucca}

"Los pintores encargados de dibujar el paisaje, deben estar, a la verdad, en las montañas, cuando tienen necesidad de que los valles se descubran bien a sus miradas; pero también únicamente desde el fondo de los valles pueden ver bien en toda su extensión las montañas y elevados sitios. Sucede lo propio en la política: si para conocer la naturaleza de los pueblos es preciso ser príncipe, para conocer la de los principados, conviene estar entre el pueblo" (Maquiavelo, 1946: 14).

\section{Bibliografía}

Aboy Carlés, G. (2001). Las dos fronteras de la democracia argentina: la reformulación de las identidades políticas de Alfonsín a Menem. Rosario: Homo Sapiens Ediciones.

Aguilar Villanueva, L. F. (1998). El futuro del Estado Nación. Revista Argentina de Ciencia Política, 2.

Anderson, J. (1995). "The exaggerated death of the nation-state" en A global world? ReOrdering Political Space. J. Anderson, C. Brook y A. Cochrane (ed). Oxford. Oxford University Press.

Anderson, P. (1998). El origen de la posmodernidad. Barcelona: Anagrama.

Arditi, B. (2000). El reverso de la diferencia. Identidad y política. Venezuela: Nueva sociedad.

Arfuch, L. (2005). Identidades, sujetos y subjetividades. 2da Edición. Buenos Aires: Prometeo Libros.

Argumedo, A. (1993). Los silencios y las voces en América Latina. Notas sobre el pensamiento nacional y popular. Buenos Aires. Ediciones del Pensamiento Nacional.

Badiou, A. (1990). ¿Se puede pensar la política? Buenos Aires: Nueva Visión.

Bauman, Z. (2003). Modernidad Líquida. Argentina: FCE.

Bauman, Z. (2005). Identidad. Buenos Aires: Losada Editorial.

Beck, U. (1998). La sociedad del riesgo. España: Paidos.

Berman, M. (1988). Todo lo sólido se desvanece en el aire. México: Siglo XXI editores.

Chuche, D. (1996). La noción de cultura en las ciencias sociales. Buenos Aires: Nueva Visión.

Ferry, J-M. (1995). "Las transformaciones de la publicidad política" en AA. VV. El nuevo espacio público. Barcelona: Editorial Gedisa.

Francais, A. (2000). El Crepúsculo del estado-nación. Una interpretación histórica en el contexto de la globalización. MOST. Documentos de debate, 47.

Fraser, J. (1992). El muro de cristal. Ideas representativas sobre el tiempo en el pensamiento occidental. Revista Archipiélago, (10), 17-54.

Giddens, A. (1995). Modernidad e identidad del yo. Barcelona: Ediciones Península. 
Giddens, A. (2001). Consecuencias de la Modernidad. España: Alianza Editorial.

Habermas, J. (2000). La constelación posnacional. España: Paidos.

Hobsbawm, E. (1994) “Identidad”. Revista Internacional de Filosofia Politica, 3, 5-17.

Laclau, E. (2000). Nuevas Reflexiones sobre la revolución de nuestro tiempo. 2da Edición. Argentina: Nueva Visión.

Laclau, E. (1997). Deconstrucción, Pragmatismo y Hegemonía. ÁGORA, 6, 63-89.

Lash, S. (1997). Sociología del postmodernismo. Buenos Aires: Amorrortu Editores.

Létourneau, J. (1997). Mundialización e identidad histórica de la región. Revista Ciclos en la historia, la economía y la sociedad. VII, VII (12), 43-57.

Maffesoli, M. (2000). "Identidad e identificación en las sociedades contemporáneas" En Arditi, B. (Ed.). El reverso de la diferencia. Identidad y política. Venezuela: Nueva sociedad.

Mann, M. (1997) "Has globalization ended the rise and rise of the Nation-Satate? Disponible en http://www.sscnet.ucla.edu/soc/faculty/mann/Doc2.pdf

Miguel. L. F. (2003). "Representação política em 3D. Elementos para uma teoria ampliada da representação política". Revista Brasileira de Ciências Sociais. 18 (51).

Ortiz, R. (1996). Otro Territorio. Ensayos sobre el mundo contemporáneo. Quilmes: Editorial de la Universidad Nacional de Quilmes.

Patrón, P. (1998). “Democracia y nueva articulación entre privado y público. El problema de los 'espacios públicos", en R. Urzua y F. Arguero (comp.) Fracturas en la gobernabilidad democrática. Chile: Centro de Análisis de Políticas Públicas de la Universidad de Chile.

Taylor, Ch. (1993). El multiculturalismo y la política del reconocimiento. México: FCE.

Vattimo, G. (1990). El fin de la modernidad. España Editorial: Gedisa.

Yannuzzi, M. A. (1997). La crisis del Estado-nación. Revista KAIROS, 1 (1), sin numeración.

Recepción: 29/10/2021

Evaluado: 26/11/2021

Versión Final: 13/12/2021 